\title{
Pump-to-Signal Transfer of Low-Frequency Intensity Modulation in Fiber Optical Parametric Amplifiers
}

\author{
Michel E. Marhic, Senior Member, IEEE, Member, OSA, Georgios Kalogerakis, Student Member, IEEE, \\ Kenneth Kin-Yip Wong, Student Member, IEEE, and Leonid G. Kazovsky, Fellow, IEEE, Fellow, OSA
}

\begin{abstract}
This paper describes the theoretical and experimental investigation of the transfer of low-frequency intensity modulation (IM) from pump to signal in fiber optical parametric amplifiers (OPAs). It is first established that low-frequency IM of the pump remains unchanged over the length of the amplifier in spite of the presence of parametric gain. The pump-power dependence of the OPA gain is then used to calculate the instantaneous effect of pump IM on the signal and idler output powers. These calculations are performed for both one- and two-pump OPAs. The main predictions are that 1 ) the ratio $\rho$ of the signal intensity modulation depth to that of the pump varies across the OPA gain spectrum and 2) for a 20-dB gain, $\rho$ can exceed 10 at some wavelengths, which indicates that this effect can be detrimental. Experiments have been performed to verify these predictions. Using sinusoidal IM of the pump, the resulting amplified signal IM was measured, and the experimental results were found to be in good agreement with the theoretical predictions.
\end{abstract}

Index Terms-Four-wave mixing (FWM), optical amplifiers, optical fibers, relative intensity noise (RIN), parametric amplification.

\section{INTRODUCTION}

A $S$ the performance of fiber optical parametrics amplifiers (OPAs) improves, it is becoming important to fully understand the mechanisms that can degrade the quality of amplified signals. The OPA optical noise figure (NF) has been reduced by effective removal of pump ASE by filtering [1] and is now below $4 \mathrm{~dB}$ [2], [3]. Optical NF, however, is not the only concern in optical communication. In particular, in nonlinear amplifiers such as Raman amplifiers, the relative intensity noise (RIN) of the pump instantaneously modulates the gain and is thus transferred to the amplified signal. In fiber OPAs, it has been shown that the inevitable Raman gain causes pump intensity modulation (IM), which is transferred to the signal, thereby increasing electrical NF [3], [4].

In fiber OPAs, pump-induced stimulated Brillouin scattering (SBS) must be suppressed. This is generally done by phase modulation (PM) or frequency modulation (FM) of the pump, resulting in pump spectra that are several gigahertz wide. FM is obtained by modulating the current of the laser diode supplying the single-frequency pump; unfortunately, this is always accompanied by inevitable pump IM. PM is obtained by using an external lithium niobate phase modulator. Ideally, phase modulators are free of IM, but in reality some amount of IM is always

Manuscript received May 12, 2004; revised October 1, 2004. This work was supported by the National Science Foundation under Grant ANI-0123441.

The authors are with the Department of Electrical Engineering, Stanford University, Stanford, CA 94305 USA (e-mail: marhic@wdm.stanford.edu).

Digital Object Identifier 10.1109/JLT.2004.840005 present. It is due to interferometric effects caused by reflections from various elements, possibly including the ends of the modulator crystal itself.

The presence of pump FM and residual IM is a significant cause for concern, because we expect that they will modulate signal and idler gains, thereby degrading performance. The effect of pump FM has been recently investigated [5], [6]. This paper explicitly investigates the pump-to-signal transfer of low-frequency IM in a fiber OPA, which generally accompanies pump FM.

It is first established that the low-frequency IM of the pump remains unchanged over the length of the amplifier in spite of the presence of parametric gain. This invariance allows the use of the well-known OPA gain expressions assuming constant pump power. Analytical expressions are then derived for the magnification transfer coefficient for low-frequency IM $\rho$ for both oneand two-pump OPAs. It is found that for an OPA with a maximum gain of $20 \mathrm{~dB}, \rho$ varies across the gain bandwidth: from 2 to 13 for a one-pump OPA and from 4 to 10 for a two-pump OPA. The paper then describes experiments, using low-frequency sinusoidal modulation of the pump intensity, which were performed to verify the theoretical predictions. Good agreement was found between the theoretical and experimental values.

\section{THEORY}

Our approach to calculating $\rho$ is to use the well-known expressions for OPA gain in the absence of pump depletion. These can be written in terms of sinh or cosh functions, and they depend explicitly on the pump power. Thus, it is a simple matter to analytically calculate the signal output power variation for a given change in pump power, which yields the IM magnification coefficient $\rho$.

For this approach to be correct as described, however, the pump IM must remain constant along the amplifier, since the basic gain expressions assume that pump power does not change along the amplifier. It is well known, however, that pump IM can grow under certain circumstances, due to modulation instability (MI) [7]. Equivalently, MI can be understood in terms of parametric amplification of modulation sidebands by a carrier acting as a pump. Clearly, if MI were to cause significant growth of IM under the conditions of interest here (low modulation frequency), then the method proposed previously for calculating $\rho$ would break down, as the usual OPA gain expressions would not be valid.

In the following section, we establish that low-frequency pump IM does not grow, even though each modulation sideband experiences parametric gain. We prove this separately for oneand two-pump OPAs. We then proceed with the calculation of $\rho$ based on the usual OPA gain expressions. 


\section{A. Evolution of Pump Amplitude Modulation in Fiber OPAs}

1) One-Pump OPA: To simplify the analysis, we assume that the initial pump IM is not accompanied by frequency modulation (FM); in that case, IM is equivalent to amplitude modulation (AM). We thus consider an OPA with a pump that at the input has sinusoidal AM at the frequency $f_{m}$. This can be viewed as the superposition of the following three waves: a carrier with real amplitude $A_{p 0}$ (average power $P_{0}=\left(A_{p 0}\right)^{2}$ ); a signal sideband with real amplitude $A_{s 0}$ at $f_{m}$ from the carrier (signal power $P_{s 0}=\left(A_{s 0}\right)^{2}$ ); and an idler sideband with real amplitude $A_{i 0}=A_{s 0}$ at $-f_{m}$ from the carrier. In the small-signal limit, the carrier power remains unchanged throughout the OPA. The carrier acts as a constant pump, providing parametric gain for the two sidebands. The output amplitudes of the signal and idler are then given by the OPA output field expressions

$$
\begin{aligned}
A_{s}(L)= & \left\lfloor A_{s 0} \cosh (g L)+\frac{i}{g}\left(\frac{\kappa}{2} A_{s 0}+r A_{i 0}^{*}\right) \sinh (g L)\right\rfloor \\
& \times \exp \left\lfloor-i\left(\frac{\kappa}{2}-2 \gamma P_{0}\right) L\right\rfloor \\
A_{i}(L)= & {\left[A_{i 0} \cosh (g L)+\frac{i}{g}\left(\frac{\kappa}{2} A_{i 0}+r A_{s 0}^{*}\right) \sinh (g L)\right] } \\
& \times \exp \left[-i\left(\frac{\kappa}{2}-2 \gamma P_{0}\right) L\right\rfloor
\end{aligned}
$$

where for a one-pump OPA: $g^{2}=r^{2}-(\kappa / 2)^{2} ; r=\gamma P_{0}$; $\kappa=\Delta \beta+2 \gamma P_{0} ; \gamma$ is the fiber nonlinearity coefficient; $\Delta \beta=$ $\beta_{s}+\beta_{i}-2 \beta_{p}$ is the wavevector mismatch, where $\beta_{l}$ is the wavevector of the $l$ th wave; $L$ is the fiber length; and $P_{0}$ is the pump power. Because of the conditions on $A_{s 0}$ and $A_{i 0}$, these two equations yield the same result, i.e.,

$$
\begin{aligned}
A_{s}(L)= & A_{i}(L) \\
= & A_{s 0}\left\lfloor\cosh (g L)+\frac{i}{g}\left(\frac{\kappa}{2}+r\right) \sinh (g L)\right\rfloor \\
& \times \exp \left\lfloor-i\left(\frac{\kappa}{2}-2 \gamma P_{0}\right) L\right\rfloor .
\end{aligned}
$$

Considering relatively small modulation frequencies, $\Delta \beta \approx 0$ (see the Appendix for justification), $g \approx 0, \kappa \approx 2 \gamma P_{0}$, and hence, $A_{s}(L)=A_{i}(L) \approx A_{s 0}(1+2 i \phi) \exp (i \phi)$, where $\phi=$ $\gamma P_{0} L$. This shows that signal and idler amplitudes grow linearly with distance. On that basis, we might expect the AM to grow at the same rate. This, however, is not true, as shown by the following argument.

Since we assume that $\Delta \beta \approx 0$, we can use the exact solution of the nonlinear Schrödinger equation for the total field, i.e.,

$$
A(L, t)=A\left(0, t^{\prime}\right) \exp \left[i \gamma L\left|A\left(0, t^{\prime}\right)\right|^{2}\right]
$$

where $t^{\prime}=t-L / v, v$ is the speed of propagation of light in the fiber, and

$$
\begin{aligned}
A\left(0, t^{\prime}\right) & =A_{p 0}+A_{s 0} e^{i \omega_{m} t^{\prime}}+A_{i 0} e^{-i \omega_{m} t^{\prime}} \\
& =A_{p 0}+2 A_{s 0} \cos \left(\omega_{m} t^{\prime}\right)
\end{aligned}
$$

is the total input field, which is real. Since the exponential in (3) is purely a phase term, we see that the instantaneous amplitude of the total output field is exactly the same as that of the

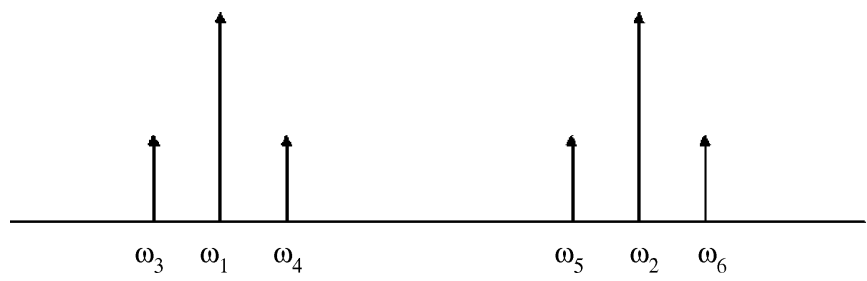

Fig. 1. Frequency assignments for the two-pump four-sideband case.

total input field (at the corresponding time). Therefore, there is no growth of pump AM along the fiber, in spite of the growth of the individual modulation sidebands. The fact that the pump IM does not grow in spite of the finite parametric gain of the individual sidebands is due to the fact that their growth corresponds to self-phase modulation (SPM), not to IM.

2) Two-Pump OPA: We now consider a two-pump OPA, with pump frequencies $\omega_{1}$ and $\omega_{2}$; pump 1 is assumed to have initial IM, as in Section II-A-1). Its modulation sidebands are then coupled to sidebands of the other pump, because nondegenerate four-wave mixing (FWM) involving the two pumps is well phase matched (see the Appendix for justification). On the other hand, we assume that the FWM interactions generating new frequency components at or near $\left(2 \omega_{1}-\omega_{2}\right)$ and $\left(2 \omega_{2}-\omega_{1}\right)$ are poorly phase matched, and we neglect these FWM terms. We thus consider the interactions between six frequencies: the two pumps, and two sidebands for each (Fig. 1). This situation has been previously investigated, in the context of MI [5]. It is known that in the limit of very low frequency modulation, $g$ vanishes and that the OPA exhibits little gain if a single sideband is present at the input. However, since IM involves the initial presence of two sidebands for one pump, we need to examine this situation closely to ascertain whether the IM grows or not, as we have done for the one-pump case.

We use a simplified model for the FWM interactions: because the modulation frequency is very small, we assume that all the linear wavevector mismatches vanish (see the Appendix for justification). Thus, we only consider phase shifts due to pump SPM and XPM, and we neglect Raman gain. The $k$ th wave is characterized by a slowly varying envelope $A_{k}, k=1-6$. The equations for the six waves are [5]

$$
\begin{aligned}
& \frac{d A_{1}}{d z}=i \gamma\left[\left(A_{1} A_{1}^{*}+2 A_{2} A_{2}^{*}\right) A_{1}+2 A_{4} A_{5} A_{2}^{*}+2 A_{4} A_{2} A_{6}^{*}\right. \\
& \left.+2 A_{3} A_{6} A_{2}^{*}+2 A_{3} A_{2} A_{5}^{*}+2 A_{3} A_{4} A_{1}^{*}\right] \\
& \frac{d A_{2}}{d z}=i \gamma\left[\left(2 A_{1} A_{1}^{*}+A_{2} A_{2}^{*}\right) A_{2}+2 A_{5} A_{4} A_{1}^{*}+2 A_{5} A_{1} A_{3}^{*}\right. \\
& \left.+2 A_{6} A_{3} A_{1}^{*}+2 A_{6} A_{1} A_{4}^{*}+2 A_{6} A_{5} A_{2}^{*}\right] \\
& \frac{d A_{3}}{d z}=i \gamma\left[2\left(A_{1} A_{1}^{*}+A_{2} A_{2}^{*}\right) A_{3}+A_{1}^{2} A_{4}^{*}\right. \\
& \left.+2 A_{1} A_{2} A_{6}^{*}+2 A_{1} A_{2}^{*} A_{5}\right] \\
& \frac{d A_{4}}{d z}=i \gamma\left[2\left(A_{1} A_{1}^{*}+A_{2} A_{2}^{*}\right) A_{4}+A_{1}^{2} A_{3}^{*}\right. \\
& \left.+2 A_{1} A_{2} A_{5}^{*}+2 A_{1} A_{2}^{*} A_{6}\right] \\
& \frac{d A_{5}}{d z}=i \gamma\left[2\left(A_{1} A_{1}^{*}+A_{2} A_{2}^{*}\right) A_{5}+A_{2}^{2} A_{6}^{*}\right. \\
& \left.+2 A_{1} A_{2} A_{4}^{*}+2 A_{1}^{*} A_{2} A_{3}\right] \\
& \frac{d A_{6}}{d z}=i \gamma\left[2\left(A_{1} A_{1}^{*}+A_{2} A_{2}^{*}\right) A_{6}+A_{2}^{2} A_{5}^{*}\right. \\
& \left.+2 A_{1} A_{2} A_{3}^{*}+2 A_{1}^{*} A_{2} A_{4}\right] \text {. }
\end{aligned}
$$


It can be shown that (5) leads to

$$
\frac{d}{d z}\left(P_{1}+P_{3}+P_{4}\right)=\frac{d}{d z}\left(P_{2}+P_{5}+P_{6}\right)=0
$$

As a result, the total field of pump 1 at $L$ must be of the form $A_{1}(L, t)=A_{1}\left(0, t^{\prime}\right) \exp \left[i \varphi_{1}(L, t)\right]$, where $\varphi_{1}(L, t)$ is a real phase. Thus, if $A_{1}\left(0, t^{\prime}\right)$ has IM with a form similar to (4), we conclude as in Section II-A-1) that $A_{1}(L, t)$ has exactly the same IM. Therefore, there is no growth of the IM of pump 1. Repeating the reasoning for pump 2, which originally has no IM, we find that it does not acquire any IM due to pump 1.

In summary, we have shown that, in a two-pump OPA, the initial IM of each pump remains unchanged over the amplifier length, as in the one-pump OPA case.

\section{B. Transfer of Pump IM to Signal IM}

Having established that pump IM remains constant along the fiber, we can now proceed with the calculation of how the variations in pump power affect the gain of a probe signal at some arbitrary wavelength in the gain bandwidth of the OPA. To simplify the notations, we now use a quasi-steady-state approach, assuming that the pump varies so slowly that we can use the usual steady-state expressions to calculate the gain. This allows us to suppress any explicit time dependence and to simply calculate changes in gain by means of derivatives. Thus, we now write that the pump power is of the form $P_{p}=P_{0}\left(1+m_{p}\right)$, where $P_{0}$ is the average power, and $m_{p}$ is the IM index. Similarly, we write the signal output power as

$$
P_{s, \text { out }}=G_{s} P_{s, \text { in }}=G_{s 0}\left(1+m_{s}\right) P_{s, \text { in }}
$$

where $P_{s, \text { in }}$ is the signal input power, free of modulation; $G_{s}$ is the signal power gain, modulated by the pump power; $G_{s 0}$ is the reference power gain, i.e., $G_{s}$ for $m_{p}=0$; and $m_{s}$ is the signal output IM index, which is same as the signal power gain modulation index. The relationship between $m_{p}$ and $m_{s}$ is obtained by expressing the fact that $G_{s}$ depends on $m_{p}$ through $P_{p}$, as follows:

$$
G_{s}=G_{s 0}+m_{p} \frac{d G_{s}}{d m_{p}}=G_{s 0}+m_{p} P_{0} \frac{d G_{s}}{d P_{p}}=G_{s 0}\left(1+m_{s}\right) .
$$

Hence, we find that the RIN magnification coefficient is

$$
\rho \equiv \frac{m_{s}}{m_{p}}=\frac{P_{0}}{G_{s 0}} \frac{d G_{s}}{d P_{p}}=\frac{P_{0}}{1+G_{i 0}} \frac{d G_{i}}{d P_{p}}
$$

where we used the fact that, in a lossless fiber OPA $G_{s}=1+G_{i}$, where $G_{i}$ is the signal-to-idler conversion gain, given by

$$
\sqrt{G_{i}}=\frac{\gamma P_{p}}{g} \sinh (g L)
$$

To calculate $\rho$, it is convenient to calculate the derivative of (10) with respect to $P_{p}$. This leads to

$$
\frac{1}{2 \sqrt{G_{i}}} \frac{d G_{i}}{d P_{p}}=\frac{\sqrt{G_{i}}}{P_{p}}+\sqrt{G_{i}}[g L \operatorname{coth}(g L)-1] \frac{1}{g} \frac{d g}{d P_{p}}
$$

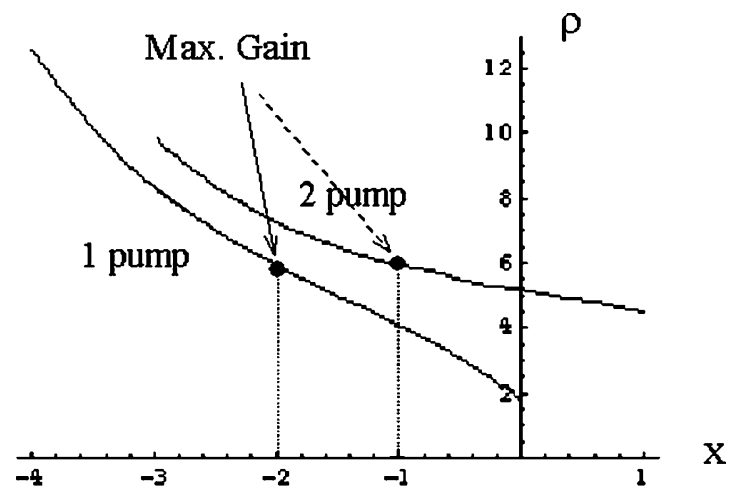

Fig. 2. Graphs of $\rho(x)$ for one- and two-pump OPAs, for $\phi=3$. Maximum gain corresponds to $x=-2$ for the one-pump case and $x=-1$ for the two-pump case, respectively.

or

$$
\frac{P_{p}}{2 G_{i}} \frac{d G_{i}}{d P_{p}}=1+[g L \operatorname{coth}(g L)-1] \frac{P_{p}}{g} \frac{d g}{d P_{p}} .
$$

Since $g^{2}=\left(\gamma P_{p}\right)^{2}-\left(\Delta \beta+u \gamma P_{p}\right)^{2} / 4$, where $u=1(2)$ for a 2(1)-pump OPA

$$
2 g \frac{d g}{d P_{p}}=\frac{\gamma^{2} P_{p}\left(4-u^{2}\right)-u \gamma \Delta \beta}{2}
$$

and

$$
\frac{P_{p}}{g} \frac{d g}{d P_{p}}=\frac{4-u(x+u)}{4-(x+u)^{2}}
$$

where $x=\Delta \beta / \gamma P_{0}$ is the ratio of linear phase shift to nonlinear phase shift. Finally,

$$
\begin{aligned}
\rho= & \frac{P_{p}}{G_{s}} \frac{d G_{i}}{d P_{p}}=\frac{G_{i}}{G_{s}} \frac{P_{p}}{G_{i}} \frac{d G_{i}}{d P_{p}} \\
= & 2 \frac{G_{i}}{G_{s}}\left\{1+[g L \operatorname{coth}(g L)-1] \frac{P_{p}}{g} \frac{d g}{d P_{p}}\right\} \\
= & \frac{2}{1+\left[\frac{g^{\prime}}{\sinh \left(g^{\prime} \phi\right)}\right]^{2}} \\
& \times\left\{1+\left[g^{\prime} \phi \operatorname{coth}\left(g^{\prime} \phi\right)-1\right] \frac{4-u(x+u)}{4-(x+u)^{2}}\right\}
\end{aligned}
$$

where $g^{\prime}=g / \gamma P_{0}=\sqrt{1-(x+u)^{2} / 4}$ is the gain coefficient normalized to its maximum value, and $\phi=\gamma P_{0} L$ is the pump nonlinear self-phase shift.

Maximum parametric gain $\left(g^{\prime}=1\right)$ is obtained for $x=-u$. For a typical OPA $\phi \approx 3$, and so $\rho \approx 2 \phi=6$. This is a nonnegligible value, which shows that in a typical fiber OPA, the pump-to-signal transfer can indeed be significant near maximum gain, which is the region of main interest.

Fig. 2 shows theoretical graphs of $\rho(\mathrm{x})$ for $\phi=3$. We see that $\rho$ can be considerably larger than 6 away from maximum gain: for a one-pump OPA, $\rho$ varies from 2 to 13 across the gain bandwidth; for a two-pump OPA, it varies from 4 to 10 . This strong wavelength dependence may have implications for the design of practical fiber OPAs. 


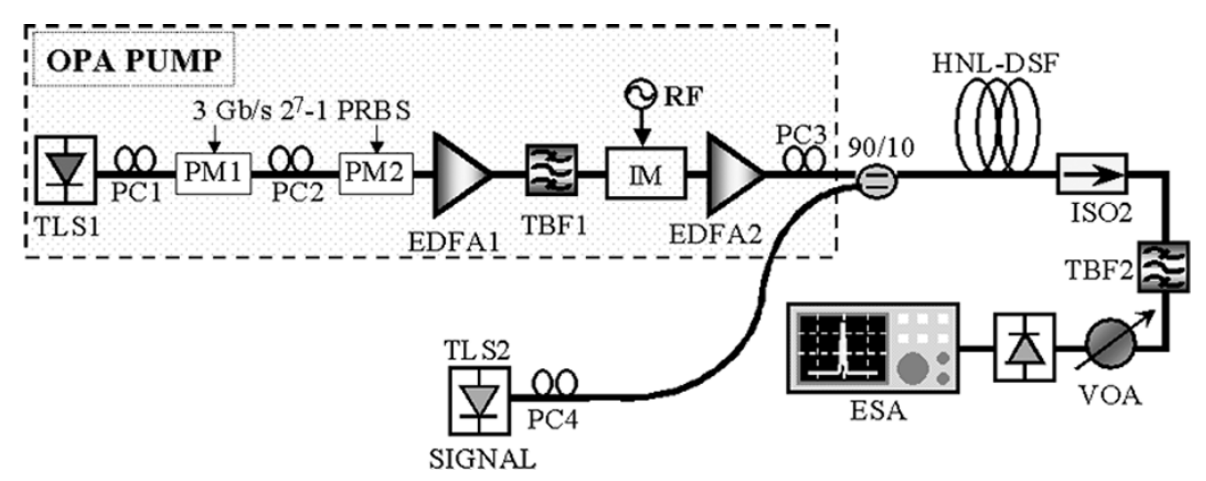

Fig. 3. One-pump OPA experimental setup. TLS: tunable laser source; PM: phase modulator; PC: polarization controller; TPF: tunable bandpass filter; IM: intensity modulator; HNL-DSF: highly nonlinear dispersion-shifted fiber; ISO: isolator; VOA: variable optical attenuator; ESA: electrical spectrum analyzer.

\section{EXPERIMENTS}

\section{A. One-Pump OPA}

We have performed experiments to verify these predictions. The experimental setup for the one-pump OPA case is shown in Fig. 3. A tunable laser serves as a pump source, and it is phase-modulated to suppress SBS. We investigate the RIN transfer effects by using an intensity modulator, which is driven by a sinusoidal radio-frequency ( $R F$ ) signal; the modulation index is 0.1 . The pump is then amplified to the desired power level by a two-stage erbium-doped fiber amplifier (EDFA2). A 10/90 coupler combines the pump and the signal into a $0.5-\mathrm{km}$-long highly nonlinear dispersion-shifted fiber (HNL-DSF) with the parameters $\lambda_{0}=1561.1 \mathrm{~nm}$, $\gamma=17 \mathrm{~W}^{-1} \mathrm{~km}^{-1}$, and $d D / d \lambda=0.03 \mathrm{ps} \mathrm{nm}^{-2} \mathrm{~km}^{-1}$. The average pump power at the input of the HNL-DSF is $25 \mathrm{dBm}$. In order to determine $\rho$, we measure the modulation index of the amplified signal at the output and compare it with the modulation index of the pump measured at the input of the HNL-DSF. The modulation index of the pump at the output of the HNL-DSF was measured to be the same as that at the input, which agrees with the theoretical analysis of Section II. Fig. 4(a) shows $\rho$ versus the modulation frequency of the pump. Experimental results agree with our theoretical model of Section II-B. The small dispersion and the small fiber length account for the fact that the RIN transfer characteristic for this OPA extends over several gigahertz, unlike for distributed Raman amplifiers where the RIN transfer characteristic extends only from hundreds of kilohertz (counterpropagating pump case) to tens of megahertz (copropagating pump case) [9]. The dip in the magnification factor as the pump modulation nears $10 \mathrm{GHz}$ is due to the limited bandwidth of the electronic components used in the experiment. In Fig. 4(b), $\rho$ versus signal wavelength is shown, together with the gain profile of the amplifier. The modulation frequency of the pump is $2 \mathrm{GHz}$ in this case. Theoretically predicted values are in good agreement with experimental data for $\rho$. The discrepancy between the theoretical and experimental gain profiles is attributed to zero-dispersion wavelength variations along the HNL-DSF.

\section{B. Two-Pump OPA}

The experimental setup for the two-pump OPA case is shown in Fig. 5. Two pump laser sources at 1574.8 and $1549.05 \mathrm{~nm}$ are combined, and they are phase-modulated by two cascaded

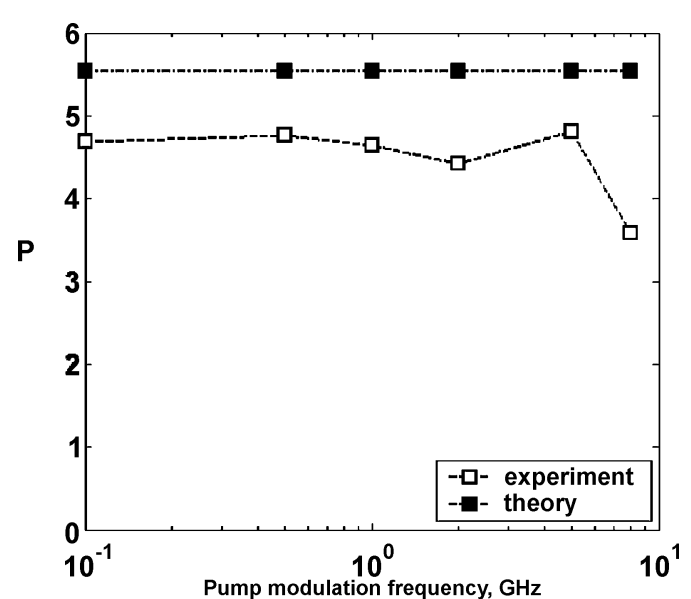

(a)
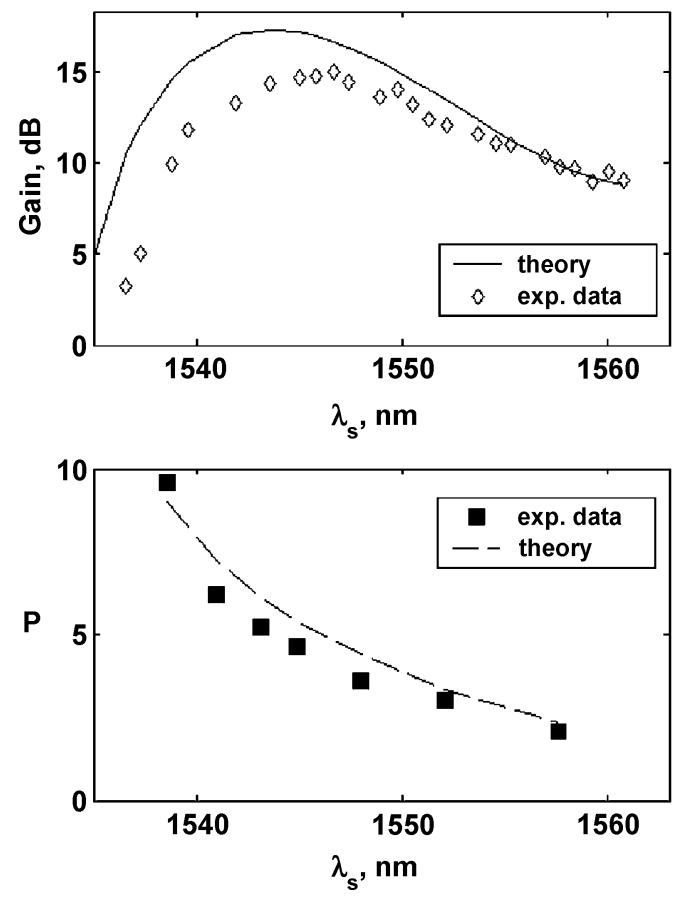

(b)

Fig. 4. (a) RIN magnification factor versus RF modulation frequency (signal wavelength is $1544.86 \mathrm{~nm}$ ). Black rectangles correspond to simulation data points using OptSim. (b) Wavelength dependence of RIN magnification factor (RF modulation frequency is $2 \mathrm{GHz}$ ).

phase modulators driven by $2^{7}-1$ pseudorandom bit sequences (PRBSs) at $3 \mathrm{GHz}$ to suppress SBS. The longer wavelength 


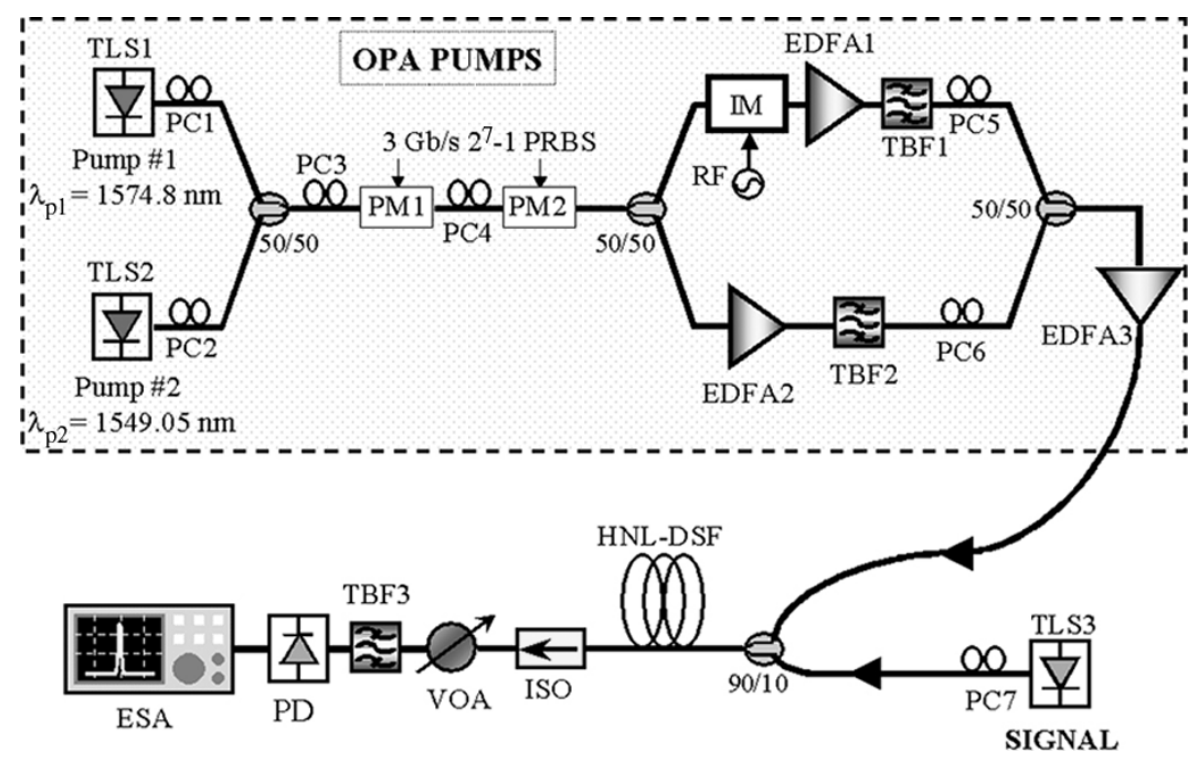

Fig. 5. Two-pump OPA experimental setup.

pump (pump 1) is intensity-modulated at $100 \mathrm{MHz}$, and both pumps are combined and amplified by a high-power EDFA. At the input of the HNL-DSF, the average power of each pump is $21.6 \mathrm{dBm}$. The pump polarization states are aligned using polarization controllers PC5 and PC6. The tunable laser source TLS3 serves as a probe signal. PC7 aligns the polarization state of the signal with that of the pumps. Signal and pumps are fed into the HNL-DSF through a 10/90 coupler. The HNL-DSF is the same as the one used in the one-pump OPA experiment. The modulation index of pump 1 at the input of HNL-DSF was $3.53 \%$. Its modulation index at the output of the HNL-DSF was measured to be $3.43 \%$. Theory indicates that there should not be a strong dependence of the intensity noise magnification factor on the modulation index of the pump, as long as that modulation index is relatively small. In the two-pump OPA case, we use a different modulation index to verify this $(3.53 \%$, instead of $10 \%$ in the one-pump case). Pump 2 is initially unmodulated, but it also acquires IM, with a modulation index of $0.91 \%$; it is due to Raman crosstalk, and it is out of phase with respect to the modulation of pump 1 [10], because Raman interaction transfers energy from the shorter- to the longer-wavelength pump. The average power of pump 2 at the output of the fiber is $0.84 \mathrm{~dB}$ lower than the average power of pump 1 (this slight change in pump power along the amplifier has not been taken into account in the calculation of $\rho$ ). $\rho$ for the two-pump OPA was measured similarly to the previous case: the ratio of the modulation index of the amplified signal at the output of the HNL-DSF over the modulation index of the total pump power at the input of the OPA. The gain profile and $\rho$ versus wavelength are shown in Fig. 6. The experimental values agree well with the theoretical predictions, especially in the high-gain region. The equations derived in Section II-B were used for the theoretical curves. These neglect any FWM components in the sidebands located in the outer spectral ranges of the two-pump wavelengths, and, therefore, they are not very accurate when the signal is located close to the pump, as can be observed in Fig. 6. $\rho$ for a two-pump OPA exhibits, in general, a smaller wavelength dependence than $\rho$ for a one-pump OPA, mainly because of the flatter gain profile of the former.

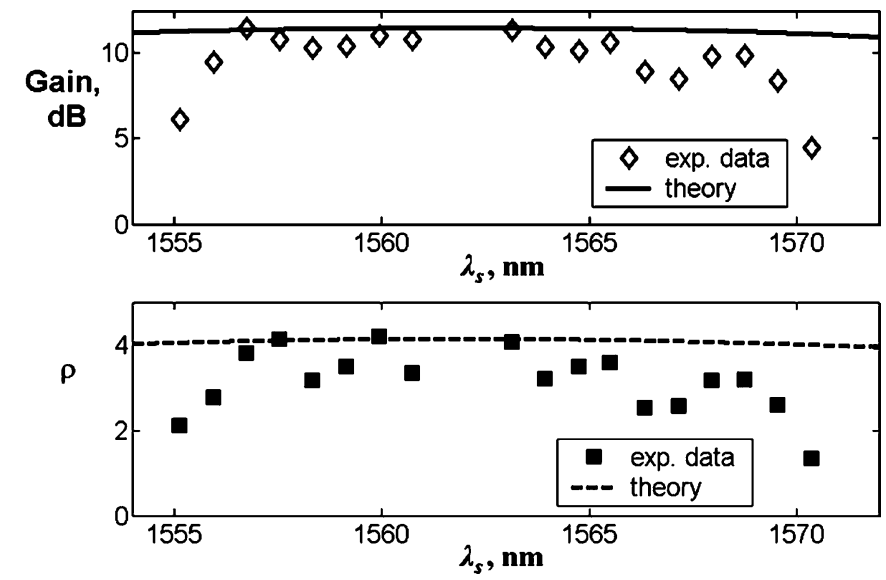

Fig. 6. Wavelength dependence of gain and RIN magnification factor in a two-pump OPA (RF modulation frequency is $100 \mathrm{MHz}$ ). Theoretical curves are obtained from the equations in Section II-B.

\section{CONCLUSION}

SBS suppression in fiber OPAs requires that the pump(s) be spectrally broadened by FM or PM. This, however, is generally accompanied by pump IM, which in turn modulates signal and idler output powers. In this paper, the transfer of IM from the pump to the signal has been investigated, and it has been shown that it occurs with a magnification factor $\rho$, which depends on the signal wavelength, and can be larger than 10 for an OPA with 20-dB maximum gain. This is true for both one- and two-pump fiber OPAs. The theoretical predictions have been experimentally verified, with good accuracy. The results of this work indicate that pump IM can be a concern in optical communication systems. The theoretical expressions for $\rho$ presented here can be used to determine the maximum pump IM tolerable in communication systems.

\section{APPENDIX}

This Appendix justifies assumptions made in Section II, namely that the wavevector mismatches entering the relevant 
OPA and FWM equations are negligible for low pump IM frequencies. The same HNL-DSF are considered as those in the experiments, with $d D / d \lambda=0.03 \mathrm{ps} \mathrm{nm}^{-2} \mathrm{~km}^{-1}$, $\beta^{(4)}=-5 \times 10^{-56} \mathrm{~s}^{4} \mathrm{~m}^{-1}$, and $L=500 \mathrm{~m}$.

Beginning with the two-pump OPA case, referring to (5) and Fig. 1 , there are two types of $\Delta \beta$ 's to consider associated, respectively, with 1) nondegenerate FWM terms, involving two frequencies near each pump, e.g., $\Delta \beta_{1245}=\beta_{4}+\beta_{5}-\beta_{1}-\beta_{2}$ and 2) degenerate FWM terms, involving three frequencies near one pump, e.g., $\Delta \beta_{1134}=\beta_{3}+\beta_{4}-2 \beta_{1}$.

Generally, the pump frequencies are adjusted so that the center frequency $\omega_{\mathrm{c}}=\left(\omega_{1}+\omega_{2}\right) / 2$ is very close to the fiber zero-dispersion frequency. This implies that $\Delta \beta_{1245}$ is dominated by the fourth-order chromatic dispersion coefficient. Following [12]

$$
\begin{aligned}
\Delta \beta_{1245} & \approx \frac{\beta^{(4)}\left[\left(\omega_{4}-\omega_{c}\right)^{4}-\left(\omega_{1}-\omega_{c}\right)^{4}\right]}{12} \\
& \approx \frac{\beta^{(4)}\left(\omega_{1}-\omega_{c}\right)^{3} \omega_{m}}{3} .
\end{aligned}
$$

To consider a worst-case situation, we assume that the pumps are $200 \mathrm{~nm}$ apart (a very large spacing) and that $f_{\mathrm{m}}=10 \mathrm{GHz}$ (a large value). We find that $\Delta \beta_{1245} L \approx 0.01 \mathrm{rad}$, i.e., that this linear phase mismatch is very small, compared with the pump nonlinear self-phase shift $\phi=\gamma P_{0} L$, which is close to $3 \mathrm{rad}$ for an OPA with $20 \mathrm{~dB}$ of gain. Thus, neglecting terms like $\Delta \beta_{1245}$ in the basic equations is justified.

On the other hand, $\Delta \beta_{1134}$ is dominated by second-order dispersion, i.e., $\Delta \beta_{1134} \approx \beta^{(2)}\left(\omega_{1}\right)\left(\omega_{1}-\omega_{c}\right)^{2} ; \beta^{(2)}\left(\omega_{1}\right)$ is obtained from the dispersion slope. It is found that $\Delta \beta_{1134} L \approx$ 0.006 ; hence, terms like $\Delta \beta_{1134}$ can safely be neglected.

For a one-pump OPA, with the pump at $\lambda_{0}$, there is a single $\Delta \beta$ to consider, namely $\Delta \beta \approx \beta^{(4)}\left(\omega_{s}-\omega_{p}\right)^{4} / 12=$ $\beta^{(4)} \omega_{m}^{4} / 12$, where $\omega_{\mathrm{s}}$ is the modulation sideband frequency [12]. Compared with $\Delta \beta_{1245}$ given by (A1), it is seen that $\Delta \beta \ll \Delta \beta_{1245}$, because $\omega_{m} \ll\left(\omega_{1}-\omega_{c}\right)$. Hence, $a$ fortiori, this $\Delta \beta$ can be neglected as well.

\section{ACKNOWLEDGMENT}

The authors would like to thank Optoelectronics Industry Development Association (OIDA) for providing the high-power erbium-doped fiber amplifier and Sprint Advanced Technology Laboratories for the loan of equipment.

\section{REFERENCES}

[1] J. L. Blows and S. E. French, "Low-noise-figure optical parametric amplifier with a continuous-wave frequency-modulated pump," Opt. Lett., vol. 27, pp. 491-493, Apr. 2002.

[2] P. L. Voss, R. Tang, and R. Kumar, "Measurement of the photon statistics and the noise figure of a fiber-optic parametric amplifier," Opt. Lett., vol. 28, pp. 549-551, Apr. 2003.

[3] P. L. Voss and P. Kumar, "Raman-induced noise-figure limit for $\chi^{(3)}$ parametric amplifiers," Opt. Lett., vol. 29, pp. 445-447, Mar. 2004.

[4] P. Kylemark, P. O. Hedekvist, H. Sunnerud, M. Karlsson, and P. A. Andrekson, "Noise characteristics of fiber optical parametric amplifiers," $J$. Lightw. Technol., vol. 22, no. 2, pp. 409-416, Feb. 2004.
[5] A. Mussot, A. Durecu-Legrand, E. Lantz, C. Simoneau, D. Bayart, H. Maillotte, and T. Sylvestre, "Impact of pump phase modulation on the gain of fiber optical parametric amplifier," IEEE Photon. Technol. Lett., vol. 16, no. 5, pp. 1289-1291, May 2004.

[6] M. E. Marhic, G. Kalogerakis, and L. G. Kazovsky, "Pump FM to signal IM conversion in fiber OPAs," presented at the OptoElectronics Communications Conf. (OECC 2004), Yokohama, Japan, Jul. 2004, Paper $13 \mathrm{P}-87$.

[7] A. Hasegawa and W. F. Brinkman, "Tunable coherent IR and FIR sources utilizing modulational instability," IEEE J. Quantum Electron., vol. QE-16, no. 7, pp. 694-697, Jul. 1980.

[8] T. Tanemura and K. Kikuchi, "Unified analysis of modulation instability induced by cross-phase modulation in optical fibers," J. Opt. Soc. Amer. B, Opt. Phys., vol. 20, pp. 2502-2514, Dec. 2003.

[9] C. R. S. Fludger, V. Handerek, and R. J. Mears, "Pump to signal RIN transfer in Raman fiber amplifiers," J. Lightw. Technol., vol. 19, no. 8, pp. 1140-1148, Aug. 2001.

[10] M. E. Marhic, F. S. Yang, and L. G. Kazovsky, "Cancellation of stimulated-Raman-scattering cross talk in wavelength-division-multiplexed optical communication systems by series or parallel techniques," J. Opt. Soc. Amer. B, Opt. Phys., vol. 15, pp. 957-963, Mar. 1998.

[11] M. E. Marhic, Y. Park, F. S. Yang, and L. G. Kazovsky, "Broadband fiber optical parametric amplifiers and wavelength converters with low-ripple Chebyshev gain spectra," Opt. Lett., vol. 21, pp. 1354-1356, Sep. 1996.

[12] M. E. Marhic, N. Kagi, T.-K. Chiang, and L. G. Kazovsky, "Broadband fiber optical parametric amplifiers," Opt. Lett., vol. 21, pp. 573-575, Apr. 1996.

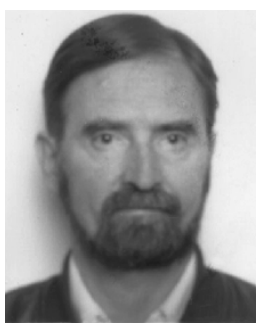

Michel E. Marhic (M'79-SM'89) received the Diplome D'Ingenieur degree from Ecole Superieure D'Electricite, France; the M.S. degree from Case Western Reserve University, Cleveland, $\mathrm{OH}$; and the Ph.D. degree from the University of California, Los Angeles (UCLA), all in electrical engineering.

He was on the faculty of the Department of Electrical Engineering at Northwestern University, Evanston, IL (1974-1998) and on sabbatical leaves at the University of South Carolina, Columbia (1979-1980) and Stanford University, Stanford, CA (1984-1985 and 1993-1994). He is currently a Consulting Professor in the Department of Electrical Engineering at Stanford University. He (co)-founded Holicon, Holographic Industries, and OPAL Laboratories. Over the past 25 years, his research has focused on several areas of applied optics, including nonlinear interactions in plasmas, optical fiber measurements, hollow infrared waveguides, holography and phase conjugation, and fiber networks. Over the past ten years, the emphasis has been on optical communication systems and on nonlinear optical interactions in fibers. He is the author or coauthor of more than 240 journal and conference papers and has been awarded eight patents.

Dr. Marhic is a Member of the Optical Society of America (OSA) and eminent Member of Tau Beta Pi. He was recipient of the Ameritech Research Professorship (1990-1991) from the Institute for Modern Communications.

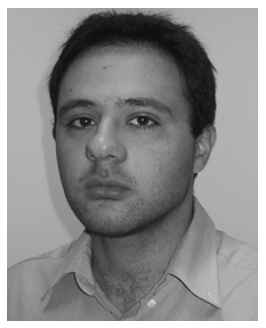

Georgios Kalogerakis (S’02) received the B.E. degree in electrical and computer engineering from National Technical University of Athens, Athens, Greece, in 2001 and the M.S. degree in electrical engineering from Stanford University, Stanford, CA, in June 2003. He is currently working toward the Ph.D. degree in electrical engineering at Stanford University.

He is a member of the Photonics and Networking Research Laboratory at Stanford University. His research focuses on fiber nonlinearity, fiber optical parametric amplifiers, and high-speed optical transmission systems. 


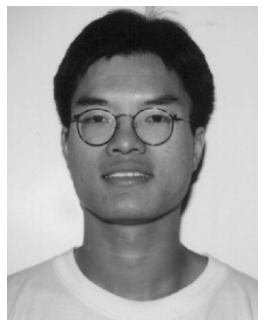

Kenneth Kin-Yip Wong (S'00) received a combined B.E. degree in electrical engineering and B.S. degree in physics from the University of Queensland, Brisbane, Australia, in 1997, the M.S. degree in electrical engineering from Stanford University, Stanford, CA, in June 1998, and the Ph.D. degree in electrical engineering at Stanford University.

$\mathrm{He}$ worked in Hewlett-Packard Laboratories as a Research Engineer and contributed to projects that included parallel optics and verticalHe also worked in Hewlett-Packard Laboratories as a Research Engineer and contributed in projects included parallel optics and vertical-cavity surface-emitting lasers (VCSELs). He is currently an Assistant Professor in the Department of Electrical and Electronic Engineering at Hong Kong University, Hong Kong. The focus of his research includes dense-wavelength-division-multiplexed (DWDM) systems, subcarrier multiplexing (SCM) optical systems, fiber nonlinearity, and fiber-optic parametric amplifiers. He is author or coauthor of more than 50 journal and conference papers.

Dr. Wong was the recipient of Optical Society of America (OSA) New Focus Student Award in 2003 and IEEE/LEOS Graduate Student Fellowship in 2003. He is the reviewer for Optics Letters, JOSA B, Optics Express, the IEEE Photonics TeChNology LetTers, the Journal of LightwaVE TECHNOLOGY, and Optics Communications.

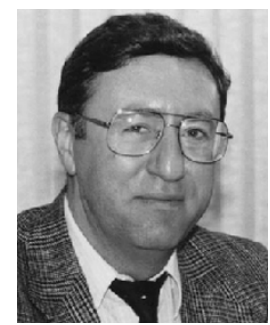

Leonid G. Kazovsky (M'80-SM'83-F'91) was with Bellcore (now Telcordia) conducting research on wavelength-division-multiplexing (WDM), high-speed, and coherent optical fiber communication systems. Since 1990, he has been a Professor of Electrical Engineering at Stanford University, Stanford, CA. After joining Stanford, he founded Optical Communication Research Laboratory (OCRL) and has led OCRL since then. While on Bellcore assignments or Stanford sabbaticals, he also worked at the Heinrich-Hertz Institute, Berlin, Germany; Hewlett-Packard Research Laboratories, Bristol, U.K.; and the Technical University of Eindhoven, The Netherlands. Through research contracts, consulting engagements, and other arrangements, he has worked with many industrial companies and U.S. Government agencies, including Sprint, DEC, GTE, AT\&T, IVP, Lucent, Hitachi, KDD, Furukawa, Fujitsu, Optivision, and Perimeter on the industrial side; and the National Science Foundation (NSF), the Defense Advanced Research Projects Agency (DARPA), the Air Force, the Navy, the Army, and BMDO on the government side. From 1998 to 1999, he took a one-year leave from Stanford University and launched a start-up company now known as Alidian Networks and serves on its Board of Directors. He has authored or coauthored two books, 150 journal technical papers, and approximately 150 conference papers.

Prof. Kazovsky is a Fellow of the Optical Society of America (OSA). He serves or has served on Editorial Boards of leading journals, such as the IEEE Transactions on Communications, the IEEE Photonics TeChNology LETTERS, and Wireless Networks, and on program committees of leading conferences, such as the Optical Fiber Communication Conference (OFC), the Conference on Lasers and Electro-Optics (CLEO), the Lasers and Electro-Optics Society (LEOS), The International Society for Optical Engineers (SPIE), and IEEE GLOBECOM. He has also served as a reviewer for various transactions, proceedings, and journals for the IEEE and the Institution of Electrical Engineers (IEE) as well as for funding agencies (such as the National Science Foundation, OFC, ERC, and the National Research Council) and for publishers (such as John Wiley \& Sons and MacMillan). 